\title{
COMPARATIVE MUCOPENETRATION ABILITY OF METRONIDAZOLE LOADED CHITOSAN AND PEGYLATED CHITOSAN NANOPARTICLES
}

\author{
SUKHBIR K ${ }^{1,2 *}$, CHAWLA V ${ }^{3}$, NARANG RK ${ }^{1}$, AGGARWAL $G^{4}$ \\ ${ }^{1}$ Department of Pharmaceutics, ISF College of Pharmacy, Moga, Punjab, India. ${ }^{2}$ Research, Innovation \& Consultancy, IK Gujral Punjab \\ Technical University, Jalandhar, Punjab, India. ${ }^{3}$ Department of Pharmaceutics, Rajiv Academy for Pharmacy, Mathura, UP, India. \\ ${ }^{4}$ Department of Pharmaceutics, Rayat and Bahra Institute of Pharmacy, Mohali, Punjab, India. Email: k_sukhbir@yahoo.co.in
}

Received: 10 February 2016, Revised and Accepted: 10 March 2017

ABSTRACT

Objective: The objective of this study is to compare the mucopenetration ability of metronidazole loaded chitosan (CS) and pegylated CS nanoparticles.

Methods: Nanoparticles were prepared by ionic gelation technique using negatively charged pH sensitive polymer, hydroxyl propyl methyl cellulose phthalate with positively charged CS and methoxy polyethylene glycol-grafted-CS (mPEG-g-CS). mPEG-g-CS was synthesized by formaldehyde linkage method and characterized by Fourier transform infrared spectroscopy. The optimized formulations were compared for morphology, particle size, polydispersity index (PDI), entrapment efficiency, bioadhesion detachment force, in vitro and in vivo mucopenetration for CS-mPEG-g-CS nanoparticles.

Results: The morphological assessment revealed smooth spherical particles with uniform dispersions. The optimized formulations particle size was found to be $202.7 \pm 27 \mathrm{~nm}$ and $294.1 \pm 46 \mathrm{~nm}$, zeta potential $26.94 \pm 2.4 \mathrm{mV}$ and $6.0 \pm 1.3 \mathrm{mV}$. PDI 0.231 and 0.268 , entrapment efficiency $79.8 \pm 5.4 \%$ and $83.6 \pm 9.7 \%$, bio-adhesion detachment force $14.98 * 10^{3} \mathrm{dyne} / \mathrm{cm}^{2}$ and $10.67 * 10^{3}$ dynes $/ \mathrm{cm}^{2}$, in vitro mucopenetration $78 \%$ and $98 \%$ for CS-mPEG-g-CS, respectively. The qualitative in vivo mucopenetration result confirms retention of fluorescein isothiocyanate (FITC) labeled mPEG-g-CS nanoparticles till 24 hrs.

Conclusion: Nanoparticles with lesser zeta potential and mucoadhesion showed higher mucosal penetration which is evident from FITC labeled histopathological mucus penetration test. Studies thus provided evidence that planned pharmaceutical strategies open new vistas for effective treatment of mucosal infections.

Keywords: Chitosan, Methoxy polyethylene glycol-grafted-chitosan, Nanoparticles, Hydroxyl propyl methyl cellulose phthalate, Ionic gelation, Mucopenetration.

(c) 2017 The Authors. Published by Innovare Academic Sciences Pvt Ltd. This is an open access article under the CC BY license (http://creativecommons. org/licenses/by/4. 0/) DOI: http://dx.doi.org/10.22159/ajpcr.2017.v10i6.17643

\section{INTRODUCTION}

Amoebiasis is a chronic disorder of intestinal mucosa with organ susceptibility to the colon. The conventional delivery systems used to treat the disease are not able to provide the drug in required concentration at target site [1]. Metronidazole is a preferred drug in the treatment of amoebiasis, giradiasis, trichomoniasis and anaerobic infections. The drug is to be delivered to the colon for its effective action against trophozoites of Entoamoeba histolytica and Giradia lamblia where the respective trophozoites reside in lumen of the caecum and large intestine and adhere to colonic mucus and epithelial layers. However, pharmacokinetic profile of metronidazole indicates that drug is completely absorbed in approximately $1 \mathrm{hr}$ after oral administration. The administration of this drug in conventional tablet dosage forms provides a minimal amount of metronidazole for local action in the colon with unwanted systemic side effects [2]. The various strategies used in the literature for colon targeting through oral route include prodrugs, enzymatic activity of colonic microflora, higher $\mathrm{pH}$, colonic pressure and transit time [3]. In addition to it, overall dimensions of the delivery system are significantly affecting the inflamed colon targeting. Nanosized particles are more effective as a delivery system for mucosal delivery due to their size and ability to protect the drug at inter and intracellular level. However, their limited permeability to mucus layer prevents the drug to reach the underlying cells.

Mucus is a viscoelastic, adhesive hydrogel protective covering of gastrointestinal tract, lungs, and female reproductive system. The thickness of intestinal mucus is $100-180 \mathrm{~nm}$, and it is composed of glycoproteins, lipids, electrolytes, cells, and cell debris [4]. The main role of mucus is to entrap foreign particles efficiently and remove them rapidly. The viscoelasticity and adhesive property of mucus retards nanoparticles diffusion in the mucus. Thus, surface modification of nanoparticles with polyethylene glycol (PEG) could help in reducing interaction by creating hydrophilic surface between mucus and delivery system, thus enabling unrestricted diffusion through the disturbed epithelium [5,6]. PEG is a hydrophilic and uncharged synthetic polymer, having many advantages as prolonged residence time in the body since it is not recognized by reticuloendothelial system and decreased degradation by enzymes when combined with the drug. It increases particle translocation through the mucus as well as mucosa. This hydrophilic surface provides an accelerated translocation into the leaky inflamed intestinal epithelium, which is ideal for colitis targeted drug delivery [7].

Chitosan (CS) is a cationic polysaccharide composed of randomly distributed - (1-4)-linked d-glucosamine (deacetylated unit) and $\mathrm{N}$-acetyl-d-glucosamine (acetylated unit). It is used to prepare nanoparticles as it opens tight junctions between epithelial cells, leading to an increase in paracellular permeability. It is water soluble and bioadhesive that readily attaches to negatively charge surface of mucus membrane [8]. Furthermore, CS has plenty of hydroxyl and amino groups which facilitate nanoparticles formulation through physical and chemical cross-linking [9], biodegradability [10], antimicrobial activity, good homeostatic properties, and anti-cancerous properties [11] further make this polymer an ideal choice for the formulation of nanoparticles. The main limitation of CS nanoparticles for oral delivery 
is the easy dissolution of the polymer matrix in the $\mathrm{pH}$ of the stomach by protonation of the amino groups at low $\mathrm{pH}$ values. As the polymer matrix gets dissolved, the entrapped drug is rapidly released in the stomach [12]. Hence, to protect the drug from stomach ionic gelation of CS was done with hydroxyl propyl methyl cellulose phthalate (HPMCP). The pKa of the free carboxylic groups of HPMCP is approximately 5.2. This free carboxylic acid becomes negatively charged at higher $\mathrm{pH}$ values and represents the driving force for the electrostatic interaction with CS.

The aim of the present study was to develop mucus penetrating, acid stable formulation of CS and methoxy PEG-grafted-CS (mPEGg-CS) nanoparticles for local delivery of metronidazole to colonic tissue by oral administration. The nanoparticles were prepared by ionic interaction of CS and MPEG-g-CS6 with HPMCP. The prepared nanoparticles were then evaluated for particle size, shape, surface charge, polydispersity index (PDI), drug loading, in vitro, ex vivo release and bioadhesion detachment force. The mucopenetration characteristics of nanoparticle were studied after oral administrations to rats by qualitative fluorescence of fluorescein isothiocyanate (FITC) labeled nanoparticles.

\section{METHODS}

Metronidazole and HPMCP HP55 grade were received ex gratia from La Pharma Pvt. Ltd. Ludhiana, Punjab, India. CS (85 deacetylated), Eudragit S100, Mono mPEG, Dialysis membrane (MW. 10-12 KDa) and FITC were procured from Sigma-Aldrich, USA. Trehalose dihydrate was obtained from Hi-Media Labs Pvt., Ltd., Mumbai. The other materials used were of analytical and pharmaceutical grades.

\section{Preparation of CS nanoparticles}

The nanoparticles were prepared with slight modification of method reported by [13] Makhlof et al., 2011. Briefly, polyelectrolyte complexation of positively charged CS with negatively charged enteric polymer HPMCP. In our preliminary investigations, polymer concentrations were optimized. For the preparation of nanoparticles, HPMCP $(0.1 \%)$ solution in $0.1 \mathrm{M}$ sodium hydroxide was slowly added to $\mathrm{CS}(0.1 \%)$ solution in $0.1 \mathrm{M}$ acetic acid containing metronidazole under magnetic stirring for 30 minutes at $100 \mathrm{rpm}$. The $\mathrm{pH}$ of the final dispersion was kept 5.5. The nanoparticles produced were collected by centrifugation at $20,000 \mathrm{rpm}(42,000 \mathrm{~g})$ for 30 minutes at $4{ }^{\circ} \mathrm{C}$, and the supernatant was used for the measurement of free metronidazole. Collected nanoparticles were washed with double distilled water, freeze at $-20^{\circ} \mathrm{C}$ in the deep freezer. Then freeze dried using D ( + ) trehalose dihydrate $(0.5 \% \mathrm{w} / \mathrm{v})$ as a cryoprotectant in lyophilizer (Martin Christ model Alpha 1-2 LD plus) at $-55^{\circ} \mathrm{C}, 0.01 \mathrm{~mm}$ of $\mathrm{Hg}$.

\section{Synthesis of pegylated CS}

Formaldehyde linking method was used to prepare copolymer of mPEGg-CS [14]. CS (85 deacetylated) (500 mg) was completely dissolved by continuous stirring in formic acid. Then, a suitable amount of mPEG (360 mg) was added. After 15 minutes, $1 \mathrm{ml}$ of formaldehyde in formic acid solution and dimethyl sulfoxide was added to above mixture and solution was allowed to stir for $12 \mathrm{hrs}$. After that, the solution was dialyzed using dialysis tube (MW. 10-12 KDa) against an excess amount of double distilled water for 3 days. The dialyzed solution was lyophilized for 3 days, and the lyophilized product was precipitated in chloroform to remove the unreacted PEG. The precipitant was dried under vacuum to obtain the product and was stored at a temperature $<4^{\circ} \mathrm{C}$ until further use.

\section{Characterization of mPEG-g-CS copolymer}

The mPEG-g-CS copolymer was characterized by Fourier transform infrared spectrophotometer (FTIR) (Thermo Nicolet-380, Thermo Scientific, U.S.A). The IR spectrum of mPEG-g-CS was recorded by placing the polymer sheet on pellet holder plate and scanning over a region of $400-4000 \mathrm{~cm}^{-1}$ (Table 1 ).

\section{Preparation of mPEG-g-CS nanoparticles}

The nanoparticles were prepared with slight modification of method reported by [13] Makhlof et al., 2011. Briefly, 0.1\% w/v mPEG-gCS was dissolved in $1 \% \mathrm{w} / \mathrm{v}$ solution of acetic acid. HPMCP $(0.1 \%)$ solution was prepared in $0.1 \mathrm{M}$ sodium hydroxide. This solution was then slowly added to mPEG-g-CS solution containing metronidazole under magnetic stirring for 30 minutes at $100 \mathrm{rpm}$. The $\mathrm{pH}$ of final dispersion was kept 5.5. The nanoparticles were then processed as described above.

\section{Characterization of nanoparticles \\ Morphology}

Morphology study of optimized formulations of nanoparticles was performed using scanning electron microscopy (SEM) (ZEISS EVO series SEM model EVO 50, Jeol, Japan). Sample was mounted on the sample holder and gold coating was done for microscopy.

\section{Particle size, PDI, and zeta potential}

Size distribution, PDI, and zeta potential of nanoparticles was determined using Zetasizer (Beckman Coulter, Delsa nano C). Samples were dispersed in double distilled water and were sonicated before estimation. All measurements were obtained in triplicate $(n=3)$.

\section{Percent drug entrapment}

Nanoparticles obtained were digested with acetic acid (2\%) solution using probe sonicator (Misonix, U.S.A.) for 20 minutes and centrifuged at $1000 \mathrm{rpm}$ for 5 minutes. The supernatant was estimated for drug concentration using ultraviolet (UV) spectrophotometer (UV-1700Pharmaspec Shimadzu, Japan) at $277 \mathrm{~nm}$. Drug entrapment (\%) of formulations was calculated using equation 1 :

$$
\begin{aligned}
& \text { Totalamt of drug- } \\
& \text { Percent Entrapment }=\frac{\text { Amt of drug present in supernatant }}{\text { Total Amt of drug }} \times 100
\end{aligned}
$$

\section{In vitro release study}

The release profile of metronidazole from CS and mPEG-g-CS polyelectrolyte complex nanoparticles were carried out in simulated gastric fluid and simulated colonic fluid (SCF) using dialysis membrane in Franz diffusion cell which was thermostatically maintained at $37^{\circ} \mathrm{C} \pm 1^{\circ} \mathrm{C}$. Each release experiment was run in triplicate. Dialysis membrane (Sigma-Aldrich, USA) was used to separate receptor and donor compartments. The donor compartment containing $2 \mathrm{ml}$ suspension of nanoparticles equivalent to $10 \mathrm{mg}$ of metronidazole, mixed for 5 seconds to aid re-suspension, in a $1 \% \mathrm{w} / \mathrm{v}$ Tween 80 solution in simulated gastric fluid/SCF [14]. Samples of $1 \mathrm{ml}$ each were withdrawn from receptor compartment at different time intervals and replaced with fresh media in equal volume. Samples were diluted where necessary, filtered and analyzed spectrophotometrically at $277 \mathrm{~nm}$.

Table 1: IR spectrum peaks of CS-mPEG-g-CS

\begin{tabular}{llll}
\hline Peaks & Chitosan & Peaks & mPEG-g-CS \\
\hline 2892 & C-H stretch & 2933 & C-H stretch increased \\
1654 & Amide I band, C-O stretch of acetyl group & 1658 & Amide I band, C-O stretch of acetyl group observed decrease \\
1577 & Amide II band, N-H stretch & 1592 & Amide II band, C-O stretch of acetyl group observed decrease \\
\hline
\end{tabular}




\section{In vitro mucopenetration study}

\section{Collection and preparation of mucus sample}

Freshly isolated pig intestinal ileum was taken from local slaughter house. It was kept in ice-cold oxygenated phosphate buffered saline (PBS) before sample processing. The colon part was taken and rinsed thoroughly with PBS. The mucus was then harvested through gentle scraping by spatula avoiding the shedding of epithelial tissue. Mucus was divided into aliquots (500 mg) and kept at $-20^{\circ} \mathrm{C}$ before experimentation [15].

\section{Mucopenetration study}

The mucus (400 mg) was equilibrated at a constant temperature of $37^{\circ} \mathrm{C}$ for 20 minutes in a vibrator to form homogenous dispersion of mucus. Then, it was placed in the donor chamber of Franz-diffusion cell with dialysis membrane located between donor and receptor compartment to support mucus. Then $2 \mathrm{ml}$ of metronidazole loaded $\mathrm{CS}$, and MPEG-g-CS nanoparticles were added to the surface of mucus. The receptor chamber was filled with PBS. After fixed interval of time $1 \mathrm{ml}$ of the sample was withdrawn and replaced with equal volume of PBS. The concentration of metronidazole was determined spectrophotometrically at $277 \mathrm{~nm}$.

\section{Synthesis of FITC conjugated CS polymer}

FITC conjugated CS was synthesized using previously reported method [16]. CS was dissolved in $20 \mathrm{ml}$ of $0.1 \mathrm{M}$ acetic acid. It was kept overnight under stirring. Then $20 \mathrm{ml}$ of methanol followed by $10 \mathrm{ml}$ of FITC solution $(2 \mathrm{mg} / \mathrm{ml}$ in methanol) were added to CS solution under continuous stirring. The reaction was allowed to proceed in dark room for $3 \mathrm{hrs}$. The FITC labeled CS was precipitated by adding $0.5 \mathrm{M}$ sodium hydroxide till a $\mathrm{pH}$ of 8-9 was obtained. It was centrifuged at $20,000 \mathrm{rpm}$ for 10 minutes. The precipitate was washed thoroughly using deionized distilled water to remove free FITC. Then FITC labeled CS was then freeze dried.

\section{Preparation of FITC labeled CS and mPEG-g-CS nanoparticles}

FITC labeled CS and mPEG-g-CS nanoparticles were prepared and as described in previous section.

\section{Bio-adhesion detachment force study}

The bio-adhesive performance of $10 \mathrm{mg}$ of CS and mPEG-g-CS nanoparticles was determined by measuring the force required to detach the formulation from the pig gastric mucosal tissue using modified [17] bio-adhesion detachment force measurement balance. The bio-adhesion detachment force $\left(\right.$ dyne $\left./ \mathrm{cm}^{2}\right)$ was calculated for nanoparticles prepared using equation 2 :

Bioadhesiveforce $=\frac{\mathrm{m}^{*} \mathrm{~g}}{\mathrm{~A}}$

Where $\mathrm{m}=$ weight in gram added to balance to detach the membrane. $\mathrm{g}=$ acceleration due to gravity in $\mathrm{m} / \mathrm{s}^{2}$.

$\mathrm{A}=$ area of the tissue exposed.

\section{In vivo mucopenetration study}

The in vivo mucopenetrationstudies were performed as per the regulation of Institutional Animal Ethical Committee of ISF College of Pharmacy, Moga, Punjab, India, vide protocol number IAEC/M17/311/2016, using FITC labeled CS and mPEG-g-CS nanoparticles. FITC labeled nanoparticles $(10 \mathrm{mg} / 2 \mathrm{ml})$ were administered using oral feeding canula to two different groups of Wistar rats $(n=12)$. After an interval of $5,8,12$, and $24 \mathrm{hrs}$, three animals were sacrificed from each group, and colon portion from each animal was excised, washed with normal saline solution, and was fixed in $10 \%$ formalin, sectioned to a thickness of $10 \mu \mathrm{m}$ and stained with eosin [18]. The fixed stained tissue sections were evaluated under digital microscope $(\times 100)$ (Motic DMWB series) using Motic Images Plus 2.0 software and inverted fluorescent microscope $(\times 40)$ (Olympus) to analyze the localization, mucoadhesion, and mucopenetration of fluorescent nanoparticles.

\section{Stability studies}

To evaluate the stability of metronidazole in the SCF, an aliquot of $10 \mathrm{mg}$ of drug was dissolved in $50 \mathrm{ml}$ of SCF and vibrated in a water bath maintained at $37^{\circ} \mathrm{C}$. Subsequently, drug estimation was carried out at 2 , 8,12 , and 24 hrs time intervals respectively, to estimate the content of metronidazole to find out the percentage degradation of the drug in the gastrointestinal tract [18].

\section{Statistical analysis}

Results are presented as mean \pm standard deviation (SD). Wherever appropriate, differences between groups were evaluated with a Student t-test (two groups) at an alpha level of 0.05 .

\section{RESULTS AND DISCUSSIONS}

The preparation of CS-HPMCP nanoparticles systems is based on an ionotropic gelation process, involving the interaction between positively charged amino groups of CS and negatively charged HPMCP to form CS-HPMCP polyelectrolyte nanoparticles, by the slow addition of anionic solutions. CS solution spontaneously changed from clear to the opalescent solution and finally to turbid suspension, indicating the formation of nanoparticles then microparticles and eventually aggregates. In a preliminary study, a pronounced increase in the particle size was observed by increasing CS concentration. Since the particle size is an important determinant of the biological efficacy of the drugloaded nanoparticles, the experimental conditions were adjusted for the preparation of nanoparticles in the size range of 200-300 nm with the highest process yield. Numbers of experiments were performed by varying the concentration of CS-HPMCP, to screen the appropriate concentration range so as to allow the formation of turbid solutions and not the aggregates. Stable and uniform nanoparticles in the size range of 200-300 nm with positive surface charge and low polydispersity were produced at a CS concentration of $0.1 \% \mathrm{w} / \mathrm{v}$ coupled with $0.1 \% \mathrm{w} / \mathrm{v}$ HPMCP while maintaining the $\mathrm{pH}$ at 5.5.

Surface coating of nanoparticles with hydrophilic material like PEG leads to reduced hydrophobicity, improved stability in mucus and enhanced drug transport across the mucus. Pegylation offers several advantages to nanoparticles like less aggregation due to steric repulsion [19]. It results in large nanoparticles, enhanced drug loading and drug penetration due to less steric hindrance between mucus and the drug transport takes place without altering the mucus structure. Indeed pegylated nanoparticles have been shown to display high permeation through cervical-vaginal mucus [5] and within sputum from cystic fibrosis patients [20].

\section{Synthesis and characterization of MPEG-g-CS}

mPEG-g-CS was characterized by the FTIR spectroscopy. The IR spectrum of the CS and mPEG-g-CS is shown in Fig. 1 and was compared with the standard IR of mPEG-g-CS. The major peaks of CS and mPEG-g-CS were compared for the conformation of grafting as in The peak at $2933 \mathrm{~cm}^{-1}$ due to $\mathrm{C}-\mathrm{H}$ stretching was increased in grafted copolymer as compared to CS $\left(2892.2 \mathrm{~cm}^{-1}\right)$. Peaks at $1658 \mathrm{~cm}^{-1}$ and $1592 \mathrm{~cm}^{-1}$ can be attributed to amide I band and amide II band, respectively. The stretching of acetyl group (C-0), decreased due to attachment of MPEG on the amide amino group [21].

\section{Characterization of nanoparticles}

CS and mPEG-g-CS nanoparticles showed mean particle size distribution between the range of $200-300 \mathrm{~nm}$ (Fig. 2) which is suitable for uptake of nanoparticles in the inflamed colon [22]. CS and mPEGg-CS nanoparticles reasonably showed monodispersion with PDI $<0.3$ (Table 2).

CS nanoparticles showed positive zeta potential of $26.94 \pm 2.4 \mathrm{mV}$, which is greater than $\mathrm{mPEG}$-g-CS nanoparticles, i.e., $6.0 \pm 1.3 \mathrm{mV}$ and is near to neutral. This may be due to the presence of PEG chains. Cationic charge 


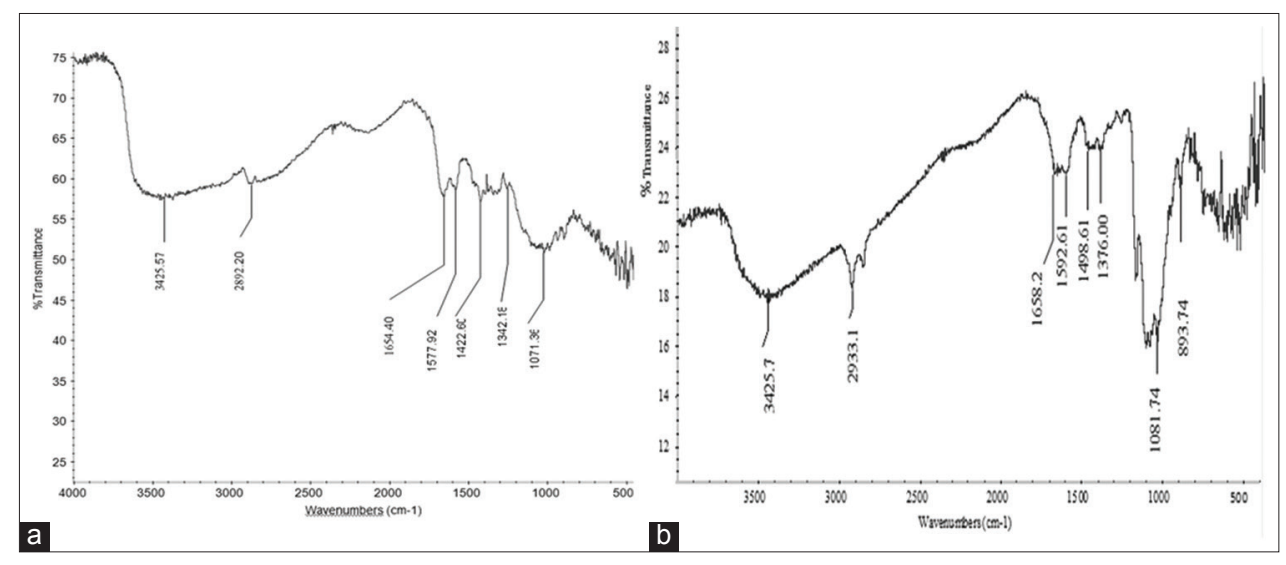

Fig. 1: Infrared spectrum of (a) Chitosan (b) poly ethylene glycol-g-chitosan

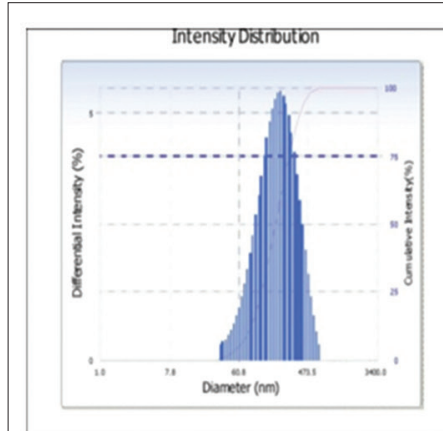

Distrbution Resuls (Contin)

\begin{tabular}{ccc}
\hline Peak & Diameter $(\mathrm{nm})$ & Std. Dev. \\
1 & 214.4 & 119.9 \\
2 & 0.0 & 0.0 \\
3 & 0.0 & 0.0 \\
4 & 0.0 & 0.0 \\
5 & 0.0 & 0.0 \\
Average & 214.4 & 119.9 \\
& & \\
Residual $:$ & $1.360 \mathrm{e} 002$ & $(0 . \mathrm{K})$
\end{tabular}

a
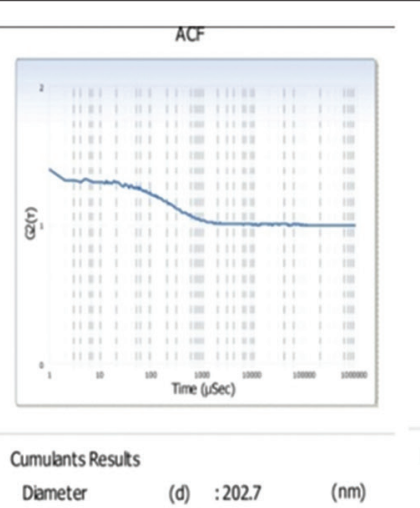

Polydispersity Index (P.L.) : 0.213

$\begin{array}{lll}\text { Difusion Const. } & \text { (D) }: 2.426 \mathrm{e}-008 \quad\left(\mathrm{~cm}^{2} / \mathrm{ser}\right.\end{array}$

Measurement Condtion

Temperaure

Temperdure

Divent Name
Refractive Index

Viscosty $: 25.0$ :WATER

$: 0.8878$

$: 11009 \quad$ (cps)

Fig. 2: Particle size and poly dispersity index of (a) chitosan and (b) methoxy poly (ethylene glycol)-grafted-chitosan nanoparticles

Table 2: Physicochemical characteristics of chitosan and mPEG-g-CS nanoparticles

\begin{tabular}{lllll}
\hline Nanoparticles & $\begin{array}{l}\text { Particle } \\
\text { size (nm) }\end{array}$ & PDI & $\begin{array}{l}\text { Zeta } \\
\text { potential } \\
\text { (mV) }\end{array}$ & $\begin{array}{l}\text { Percent drug } \\
\text { entrapment } \\
(\%)\end{array}$ \\
\hline Chitosan & $202.7 \pm 27$ & 0.213 & $26.94 \pm 2.4$ & $79.8 \pm 5.4$ \\
mPEG-g-CS & $294.1 \pm 46$ & 0.268 & $6.0 \pm 1.3$ & $83.6 \pm 9.7$ \\
\hline
\end{tabular}

mPEG-g-CS: Methoxy poly ethylene glycol-grafted-chitosan, PDI: Poly dispersity index

of CS thus prevents the interactions with mucin fibers. Both types of formulations showed very good entrapment efficiency. Comparatively, the particle size of mPEG-g-CS was more than CS nanoparticles thus entrapment efficiency was also better [23].

SEM of freeze dried CS, and mPEG-g-CS nanoparticles are shown in Fig. 3. The nanoparticles were seen as distinct spherical, consistent solid surface with porous structure. The observed matrix structure of nanoparticles was formed due to electrostatic force of attraction between anionic group of HPMCP and cationic group of CS [13].

\section{Release studies}

In vitro release studies were carried out on CS and MPEG-g-CS nanoparticles to evaluate the effect of pegylation. The dialysis method has been frequently reported for this purpose as it facilitates the separation of the released drug from the bound drug. However, the release rate of the drug is usually affected by the diffusional resistance of the membrane. In addition, sink conditions are difficult to be attained on the dialysis membrane resulting in a partitioncontrolled release profile. In the current study, metronidazole release from the CS and MPEG-g-CS nanoparticles was evaluated in simulated gastric fluid $(0.1 \mathrm{M} \mathrm{HCl}$ solution) and SCF ( $\mathrm{pH}$ 6.8). Metronidazole released from the CS nanoparticles in $2 \mathrm{hrs}$ incubation period in $0.1 \mathrm{M} \mathrm{HCl}$ solution was $16.08 \pm 3.51 \%$ while in $\mathrm{mPEG}$-g-CS nanoparticles was $13.23 \pm 3.2 \%$ (Fig. 4 ). After $2 \mathrm{hrs}$, there was a burst release of metronidazole due to the solubility of HPMCP in basic medium. When release profiles of CS and mPEG-g-CS nanoparticles were compared, pegylation led to enhanced drug release owing to increased hydrophilicity of nanoparticles by PEG. The solubility of CS in acidic medium is also reduced due to entanglement with HPMCP in polymeric matrix [12].

\section{In vitro mucus mucopenetration study}

The cumulative amount released from metronidazole loaded nanoparticles was 78\% and 98\% for CS and mPEG-g-CS, respectively. Comparing the two types of nanoparticles, there was enhanced release from mPEG-g-CS nanoparticles. This could be explained on the basis of enhanced solubility courtesy hydrophilicity of pegylated nanoparticles. The release study was conducted till $24 \mathrm{hrs}$. 

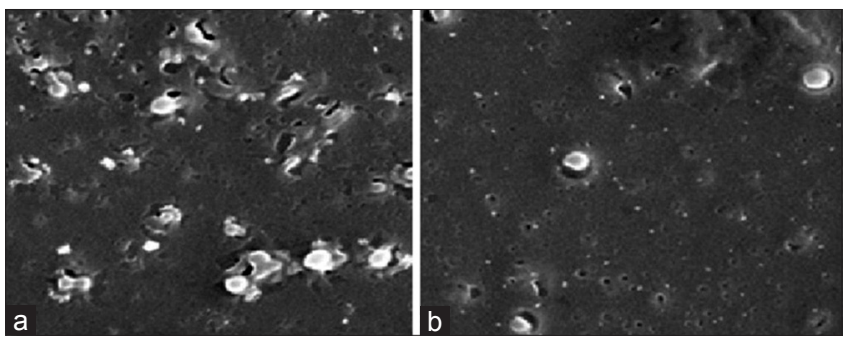

Fig. 3: Scanning electron microscopy of (a) chitosan and (b) chitosan methoxy poly (ethylene glycol)-grafted-chitosan nanoparticles

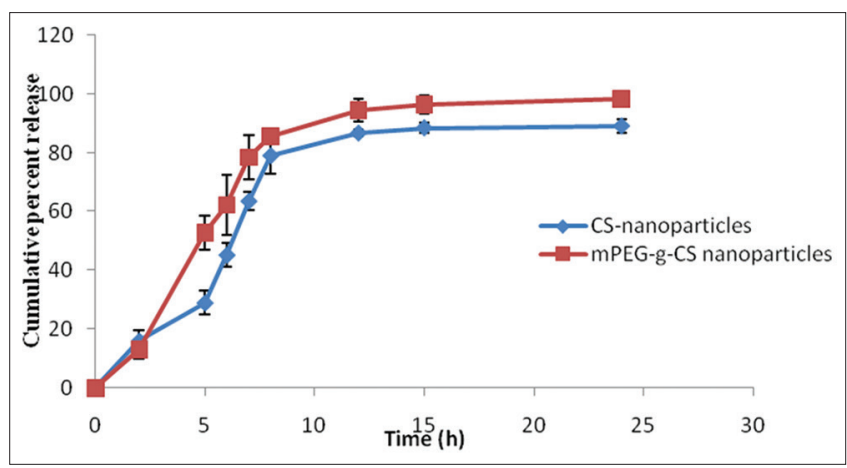

Fig. 4: Cumulative percent release from chitosan methoxy poly (ethylene glycol)-grafted-chitosan nanoparticles

\section{Bio-adhesion detachment force study}

The bioadhesion detachment force studies on nanoparticles $(n=3)$ revealed the detachment stress was up to $14.98 \times 10^{3}$ dyne $/ \mathrm{cm}^{2}$ for CS nanoparticles and it was $10.67 \times 10^{3}$ dyne $/ \mathrm{cm}^{2}$ for mPEG-g-CS nanoparticles. The cationic amino groups present on the CS interact electrostatically with mucin glycoproteins, sialic acid, and other anionic moieties present on gastric mucosa [24]. The decrease in mucoadhesive capacity of mPEG-g-CS nanoparticles can be attributed to decrease in surface amino groups by ionic interaction [6]. This decline in bioadhesion can help the nanoparticles to infiltrate at a faster rate in gastric mucosa thus proving the utility of coated nanoparticles better for enhanced penetration and accumulation at the site of infection beneath mucosa.

\section{In vivo mucopenetration study}

The FITC labeled in vivo mucopenetration studies of CS nanoparticles in Wistar rats revealed localization and internalization, i.e., mucoadhesion and mucopenetration of good number of nanoparticles up to $8 \mathrm{hrs}$ as shown in Fig. 5. The histopathology study of FITC labeled CS nanoparticles revealed that at in first 5 hrs nanoparticles got adhered to the colonic mucosa, but the penetration was visible only after $8 \mathrm{hrs}$. However, histopathological study of 24 hrs showed no fluorescence in the rat mucosa. Thus, the results reveal that the particles may be cleared with mucus as adhered and penetrated to the outer mucus layer. However, when the results were compared with FITC labeled mPEGg-CS nanoparticles, the internalization of the particles were more at 5 and 8 hrs. This may be due to the reduction of surface charge which accelerates the adhesion and penetration of the nanoparticles into the mucus. A study conducted at 12 and 24 hrs showed good number of fluorescent particles in the inner mucus layer. This could be explained by taking surface charge into consideration irrespective of the particle size. There may be a less electrostatic interaction between mucin fiber with pegylated nanoparticles which accelerates the process of penetration into the outer mucus and then into the inner mucus, proximity to the epithelial cells [1]. Furthermore, particles were not cleared with mucus and remained at the site even after the mucus clearance. In addition to improving transport, pegylation may also enhance the stability

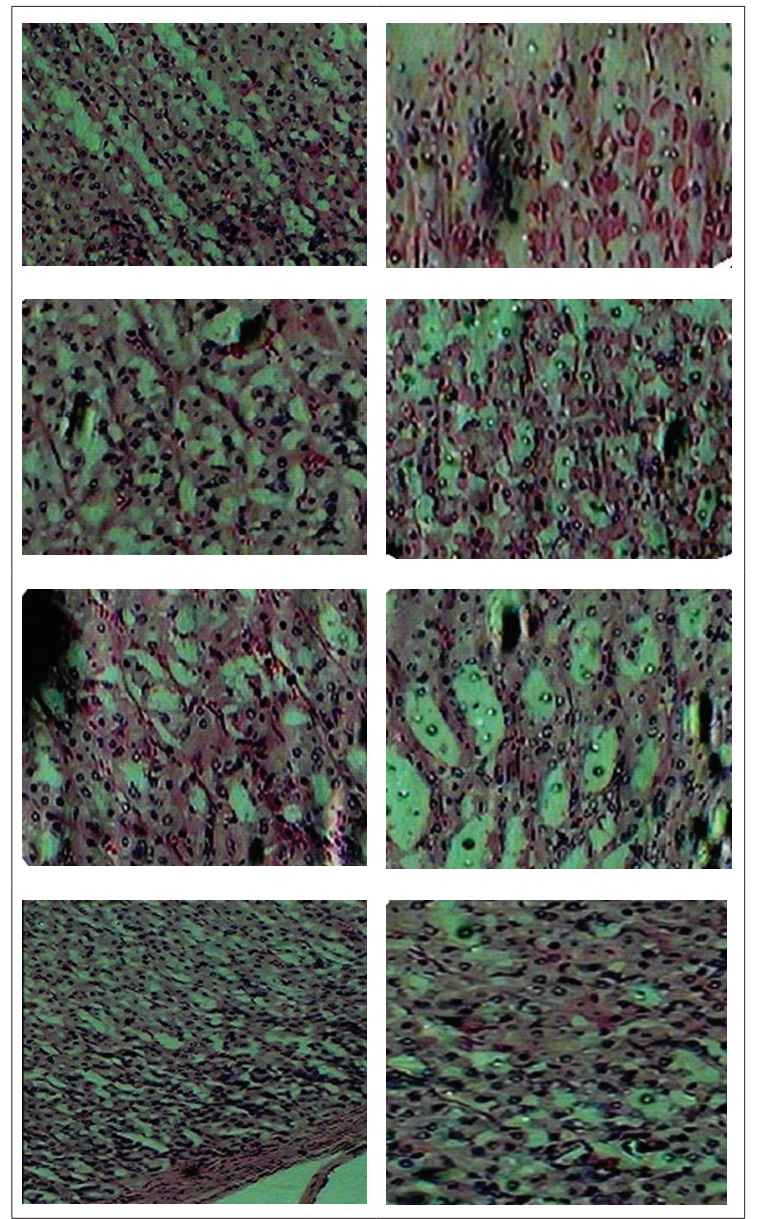

Fig. 5: Histopathological study of fluorescein isothiocyanate labeled chitosan and methoxy poly (ethylene glycol)-graftedchitosan

of nanoparticles in mucus. Stability is particularly important when particles must diffuse through a thick mucus layer to reach underlying cells. There are remote chances that the rapid transport of pegylated particles is due to alterations of the mucus structure because they do not interact significantly with mucus. Instead, particles likely move in low-viscosity channels or pores within the mucus [6]. These results confirm the novel concept of mucopenetration by modifying the surface chemistry and shielding or decreasing the cationic charge on polymers like CS to increase the motility in mucosa [5,20]. Pegylation reduces the aggregation of the particles due to steric hindrance and protects the drug from degradation [19].

\section{Stability of metronidazole in SCF}

The percentage degradation of metronidazole in SCF was found to $1.39 \pm 0.11 \%$ in 24 hrs suggested sufficient stability of the drug in SCF.

\section{CONCLUSION}

Poor mucus penetration of drug delivery system greatly reduces the therapeutic efficacy of medications used to treat mucosal infection. Proposed delivery strategies were introduced to enhance mucus penetration relying on physicochemical properties of nanoparticles. Studies inferred that physicochemical properties of drug carrier play an important role in determining the mucus penetration efficacy. Pegylation clearly enhanced the mucus penetration of nanoparticles, which is attributed to the change in surface properties particularly zeta potential, where particles with lesser zeta potential greatly reduce mucoadhesion and thus promotes muco-penetration. Studies thus provide evidence that planned pharmaceutical strategies 
open the window of opportunity for effective treatment of mucosal infections.

\section{ACKNOWLEDGMENTS}

The authors are grateful to the Chairman and Department of Pharmaceutics, ISF College of Pharmacy, Moga, Punjab, India for providing necessary facilities to complete the research work.

\section{REFERENCES}

1. Coco R, Plapied L, Pourcelle V, Jérôme C, Brayden DJ, Schneider YJ, et al. Drug delivery to inflamed colon by nanoparticles: Comparison of different strategies. Int J Pharm 2013;440(1):3-12.

2. Krishnaiah YS, Bhaskar Reddy PR, Satyanarayana V, Karthikeyan RS. Studies on the development of oral colon targeted drug delivery systems for metronidazole in the treatment of amoebiasis. Int J Pharm 2002;236:43-55

3. Hua S, Marks E, Schneider JJ, Keely S. Advances in oral nano-delivery systems for colon targeted drug delivery in inflammatory bowel disease: Selective targeting to diseased versus healthy tissue. Nanomedicine 2015;11(5):1117-32.

4. Li X, Chen D, Le C, Zhu C, Gan Y, Hovgaard L, et al. Novel mucuspenetrating liposomes as a potential oral drug delivery system: Preparation, in vitro characterization, and enhanced cellular uptake. Int J Nanomedicine 2011;6:3151-62.

5. Tang BC, Dawson M, Lai SK, Wang YY, Suk JS, Yang M, et al. Biodegradable polymer nanoparticles that rapidly penetrate the human mucus barrier. Proc Natl Acad Sci U S A 2009;106(46):19268-73.

6. Lai SK, Wang YY, Hanes J. Mucus-penetrating nanoparticles for drug and gene delivery to mucosal tissues. Adv Drug Deliv Rev 2009;61(2):158-71.

7. Lautenschläger C, Schmidt C, Lehr CM, Fischer D, Stallmach A. PEGfunctionalized microparticles selectively target inflamed mucosa in inflammatory bowel disease. Eur J Pharm Biopharm 2013;85:578-86.

8. Sadhasivam L, Dey N, Francis AP, Devasena T. Transdermal patches of chitosan nanoparticles for insulin delivery. Int J Pharm Pharm Sci 2015;7(5):84-8.

9. Ruby JJ, Pandy VP. Chitosan nanoparticles as a nasal drug delivery for memantine hydrochloride. Int J Pharm Pharm Sci 2015;7(1):34-7.

10. Bagheri KS, Taghizadeh SM, Mirzade H. An investigation on the shortterm biodegradability of chitosan with various molecular weights and degrees of deacetylation. Carbohydr Polym 2009;78:773-8

11. Gan Q, Wang T, Cochrane C, McCarron P. Modulation of surface charge, particle size and morphological properties of chitosan-TPP nanoparticles intended for gene delivery. Colloids Surf B Biointerfaces
2005;44(2-3):65-73.

12. George M, Abraham TE. Polyionic hydrocolloids for the intestinal delivery of protein drugs: Alginate and chitosan - A review. J Control Release 2006;114(1):1-14

13. Makhlof A, Tozuka Y, Takeuchi H. Design and evaluation of novel pHsensitive chitosan nanoparticles for oral insulin delivery. Eur J Pharm Sci 2011;42(5):445-51

14. Kulkarni AR, Lin YH, Liang HF, Chang WC, Hsiao WW, Sung HW. A novel method for the preparation of nanoaggregates of methoxy polyethyleneglycol linked chitosan. J Nanosci Nanotechnol 2006;6(910):2867-73.

15. Abdulkarim M, Agulló N, Cattoz B, Griffiths P, Andreas B, Borros S, et al. Nanoparticle diffusion within intestinal mucus: Three-dimensional response analysis dissecting the impact of particle surface charge, size and heterogeneity across polyelectrolyte, pegylated and viral particles. Eur J Pharm Biopharm 2015;97:230-8.

16. Qaqish RB, Amiji MM. Synthesis of a fluorescent chitosan derivative and its application for the study of chitosan-mucin interactions. Carbohydr Polym 1999;38:99-107.

17. Kerec M, Bogataj M, Mugerle B, Gasperlin M, Mrhar A. Mucoadhesion on pig vesical mucosa: Influence of polycarbophil/calcium interactions. Int J Pharm 2002;241(1):135-43.

18. Ramteke S, Ganesh N, Bhattacharya S, Jain NK. Amoxicillin, clarithromycin, and omeprazole based targeted nanoparticles for the treatment of H. pylori. J Drug Target 2009;17(3):225-34.

19. Li Y, Pei Y, Zhang X, Gu Z, Zhou Z, Yuan W, et al. PEGylated PLGA nanoparticles as protein carriers: Synthesis, preparation and biodistribution in rats. J Control Release 2001;71(2):203-11.

20. Suk JS, Lai SK, Wang YY, Ensign LM, Zeitlin PL, Boyle MP, et al. The penetration of fresh undiluted sputum expectorated by cystic fibrosis patients by non-adhesive polymer nanoparticles. Biomaterials 2009;30(13):2591-7.

21. Yang X, Zhang Q, Wang Y, Chen H, Zhang H, Gao F, et al. Selfaggregated nanoparticles from methoxy poly (ethylene glycol)-modified chitosan: Synthesis; characterization; Aggregation and methotrexate release in vitro. Colloids Surf B Biointerfaces 2008;61(2):125-31.

22. Lamprecht A, Schäfer U, Lehr CM. Size-dependent bioadhesion of micro-and nanoparticulate carriers to the inflamed colonic mucosa. Pharm Res 2001;18(6):788-93.

23. Redhead HM, Davis SS, Illum L. Drug delivery in poly (lactide-coglycolide) nanoparticles surface modified with poloxamer 407 and poloxamine 908: In vitro characterisation and in vivo evaluation. J Control Release 2001;70(3):353-63.

24. Deacon MP, McGurk S, Roberts CJ, Williams PM, Tendler SJ, Davies MC, et al. Atomic force microscopy of gastric mucin and chitosan mucoadhesive systems. Biochem J 2000;348:557-63. 